\title{
Natural Filtrations of Infinite-Dimensional Modular Contact Superalgebras
}

\author{
Qiang Mu \\ School of Mathematical Sciences, Harbin Normal University, Harbin 150025, China \\ Correspondence should be addressed to Qiang Mu; qmu520@gmail.com
}

Received 19 December 2013; Accepted 24 March 2014; Published 24 April 2014

Academic Editor: P. N. Shivakumar

Copyright (C) 2014 Qiang Mu. This is an open access article distributed under the Creative Commons Attribution License, which permits unrestricted use, distribution, and reproduction in any medium, provided the original work is properly cited.

The natural filtration of the infinite-dimensional contact superalgebra over an algebraic closed field of positive characteristic is proved to be invariant under automorphisms by characterizing ad-nilpotent elements and the subalgebras generated by certain adnilpotent elements. Moreover, we obtain an intrinsic characterization of contact superalgebras and a property of automorphisms of these Lie superalgebras.

\section{Introduction}

Filtration structures play an important role in the classification of modular Lie algebras (see $[1,2])$ and nonmodular Lie superalgebras (see $[3,4]$ ), respectively. We know that the Lie algebras and Lie superalgebras of Cartan type possess a natural filtration structure. The natural filtrations of finitedimensional modular Lie algebras of Cartan type were proved to be invariant in $[5,6]$. In the infinite-dimensional case, the same conclusion was proved in [7], by determining adnilpotent elements. In the case of Lie superalgebras of Cartan type, the invariance of the natural filtrations of some Lie superalgebras was proved in $[8,9]$. Similar results for Lie superalgebras of generalized Cartan type were obtained in [10-12], respectively.

In this paper, we consider the infinite-dimensional modular contact superalgebra $K(2 r+1, n)$, which is analogous to the one in the nonmodular situation (see [13]). But since the principal $\mathbb{Z}$-gradations of Lie superalgebras of Cartan type are different (see [13]), most results and proofs for other Lie superalgebras cannot be applied to contact superalgebras. Therefore the corresponding results and proofs for contact superalgebras have to be established separately. By determining the ad-nilpotent elements and subalgebras generated by certain ad-nilpotent elements, we prove the main result of this paper.
Theorem 1. The natural filtration of the infinite-dimensional contact Lie superalgebra is invariant under automorphisms.

Thereby, one obtains the following theorems.

Theorem 2. Suppose that $r, r^{\prime}, n, n^{\prime}$ are positive integers. Then $K(2 r+1, n) \cong K\left(2 r^{\prime}+1, n^{\prime}\right)$ if and only if $(r, n)=\left(r^{\prime}, n^{\prime}\right)$.

Theorem 3. Let $\phi, \psi$ be automorphisms of $K(2 r+1, n)$. Then $\phi=\psi$ if and only if $\left.\phi\right|_{K_{[-1]}}=\left.\psi\right|_{K_{[-1]}}$.

The paper is organized as follows. In Section 2, we recall the necessary definitions concerning the modular contact superalgebra $K(2 r+1, n)$. In Section 3, we study the adnilpotent elements of $K(2 r+1, n)$. In Section 4 , we complete the proofs of Theorems 1-3.

\section{Preliminaries}

Throughout this paper, $\mathbb{F}$ denotes an algebraic closed field of characteristic $p>2$ and $\mathbb{Z}_{2}=\{\overline{0}, \overline{1}\}$ the ring of integers modulo 2. Let $\mathbb{N}$ and $\mathbb{N}_{0}$ denote the sets of positive integers and nonnegative integers, respectively. Given $m \in \mathbb{N}, m>1$ and $\alpha=\left(\alpha_{1}, \alpha_{2}, \ldots, \alpha_{m}\right) \in \mathbb{N}_{0}^{m}$, we put $|\alpha|=\sum_{i=1}^{m} \alpha_{i}$. Let $\mathcal{O}(m)$ denote the divided power algebra over $\mathbb{F}$ with basis $\left\{x^{(\alpha)} \mid \alpha \in \mathbb{N}_{0}^{m}\right\}$. For $\varepsilon_{i}=\left(\delta_{i 1}, \delta_{i 2}, \ldots, \delta_{i m}\right), i=1,2, \ldots, m$, we 
abbreviate $x^{\left(\varepsilon_{i}\right)}$ to $x_{i}$. Let $\Lambda(n)$ be the exterior superalgebra over $\mathbb{F}$ in $n$ variables $x_{m+1}, x_{m+2}, \ldots, x_{s}$, where $s=m+n$. Denote by $\mathcal{O}(m, n)$ the tensor product $\mathcal{O}(m) \otimes \Lambda(n)$. The trivial $\mathbb{Z}_{2}$-gradation of $\mathcal{O}(m)$ and the natural $\mathbb{Z}_{2}$-gradation of $\Lambda(n)$ induce a $\mathbb{Z}_{2}$-gradation of $\mathcal{O}(m, n)$ such that $\mathcal{O}(m, n)$ is an associative superalgebra. For $g \in \mathcal{O}(m)$ and $f \in \Lambda(n)$, we abbreviate $g \otimes f$ to $g f$. For $\alpha, \beta \in \mathbb{N}_{0}^{m}$ and $i, j=m+1, m+$ $2, \ldots, s$, the following formulas in $\mathcal{O}(m, n)$ hold:

$$
\begin{gathered}
x^{(\alpha)} x^{(\beta)}=\left(\begin{array}{c}
\alpha+\beta \\
\alpha
\end{array}\right) x^{(\alpha+\beta)}, \quad x_{i} x_{j}=-x_{j} x_{i}, \\
x^{(\alpha)} x_{j}=x_{j} x^{(\alpha)},
\end{gathered}
$$

where

$$
\left(\begin{array}{c}
\alpha+\beta \\
\alpha
\end{array}\right)=\prod_{i=1}^{m}\left(\begin{array}{c}
\alpha_{i}+\beta_{i} \\
\alpha_{i}
\end{array}\right)
$$

Put $Y_{0}=\{1,2, \ldots, m\}, Y_{1}=\{m+1, m+2, \ldots, s\}$, and $Y=Y_{0} \cup Y_{1}$. Let

$$
\begin{gathered}
\mathbb{B}_{k}=\left\{\left(i_{1}, i_{2}, \ldots, i_{k}\right) \mid m+1 \leq i_{1}<i_{2}<\cdots<i_{k} \leq s\right\}, \\
\mathbb{B}(n)=\bigcup_{k=0}^{n} \mathbb{B}_{k},
\end{gathered}
$$

where $\mathbb{B}_{0}=\varnothing$. Given $u=\left(i_{1}, i_{2}, \ldots, i_{k}\right) \in \mathbb{B}_{k}$, set $|u|=k$, $\{u\}=\left\{i_{1}, i_{2}, \ldots, i_{k}\right\}$, and $x^{u}=x_{i_{1}} x_{i_{2}} \cdots x_{i_{k}}\left(|\varnothing|=0, x^{\varnothing}=1\right)$. Then $\left\{x^{(\alpha)} x^{u} \mid \alpha \in \mathbb{N}_{0}^{m}, u \in \mathbb{B}(n)\right\}$ is an $\mathbb{F}$-basis of the infinitedimensional superalgebra $\mathcal{O}(m, n)$.

Let $D_{1}, D_{2}, \ldots, D_{s}$ be the linear transformations of $\mathcal{O}(m, n)$ such that

$$
D_{i}\left(x^{(\alpha)} x^{u}\right)= \begin{cases}x^{\left(\alpha-\varepsilon_{i}\right)} x^{u} & i \in Y_{0} \\ x^{(\alpha)} \cdot \frac{\partial x^{u}}{\partial x_{i}} & i \in Y_{1}\end{cases}
$$

Then $D_{1}, D_{2}, \ldots, D_{s}$ are superderivations of the superalgebra $\sigma(m, n)$. Let

$$
W(m, n)=\left\{\sum_{i=1}^{s} a_{i} D_{i} \mid a_{i} \in \mathcal{O}(m, n), i \in Y\right\} .
$$

Then $W(m, n)$ is an infinite-dimensional Lie superalgebra contained in $\operatorname{Der}(\mathcal{O}(m, n))$ (see [14]). If $\operatorname{deg}(x)$ appears in some expression in this paper, we always regard $x$ as a $\mathbb{Z}_{2}$ homogeneous element and $\operatorname{deg}(x)$ as the $\mathbb{Z}_{2}$-degree of $x$. Then $\operatorname{deg}\left(D_{i}\right)=\mu(i)$, where

$$
\mu(i)= \begin{cases}\overline{0} & i \in Y_{0} \\ \overline{1} & i \in Y_{1} .\end{cases}
$$

The following formula holds in $W(m, n)$ (see [14]):

$$
\left[a D_{i}, b D_{j}\right]=a D_{i}(b) D_{j}-(-1)^{\operatorname{deg}\left(a D_{i}\right) \operatorname{deg}\left(b D_{j}\right)} b D_{j}(a) D_{i},
$$

where $a, b \in \mathcal{O}(m, n)$ and $i, j \in Y$.
Hereafter let $r$ be a positive integer and let $m=2 r+1$. Put $J=Y \backslash\{m\}$ and $J_{0}=Y_{0} \backslash\{m\}$. For $i \in J$, define

$$
i^{\prime}=\left\{\begin{array}{ll}
i+r & 1 \leq i \leq r \\
i-r & r<i \leq 2 r \\
i & m<i \leq s,
\end{array} \quad \sigma(i)= \begin{cases}1 & 1 \leq i \leq r \\
-1 & r<i \leq 2 r \\
1 & i \in Y_{1} .\end{cases}\right.
$$

Let $D_{K}: \mathcal{O}(m, n) \rightarrow W(m, n)$ be the linear mapping such that

$$
D_{K}(f)=\sum_{i=1}^{s} f_{i} D_{i}
$$

where

$$
\begin{gathered}
f_{i}=(-1)^{\mu(i) \operatorname{deg}(f)}\left(x_{i} D_{m}(f)+\sigma\left(i^{\prime}\right) D_{i^{\prime}}(f)\right), \quad \forall i \in J, \\
f_{m}=2 f-\sum_{i \in J} x_{i} D_{i}(f) .
\end{gathered}
$$

Then

$$
\left[D_{K}(f), D_{K}(g)\right]=D_{K}(\llbracket f, g \rrbracket)
$$

where $\llbracket f, g \rrbracket=D_{K}(f)(g)-2 D_{m}(f) g$ (see [14]). It follows directly from (11) and the injectivity of $D_{K}$ that [, \ defines a Lie multiplication on $\mathcal{O}(m, n)$. This Lie superalgebra, denoted by $K(2 r+1, n)$, is called the infinite-dimensional contact superalgebra. In the sequel, we simply write $K$ for $K(2 r+1, n)$.

The following formula holds in $K$ (see [14]):

$$
\begin{aligned}
\llbracket f, g \rrbracket=\sum_{i \in J} \sigma(i)(-1)^{\mu(i) \operatorname{deg}(f)} D_{i}(f) D_{i^{\prime}}(g) \\
\quad+\left(2 f-\sum_{i \in J} x_{i} D_{i}(f)\right) D_{m}(g) \\
\quad-(-1)^{\operatorname{deg}(f) \operatorname{deg}(g)}\left(2 g-\sum_{i \in J} x_{i} D_{i}(g)\right) D_{m}(f) .
\end{aligned}
$$

Then $K=\bigoplus_{i=-2}^{\infty} K_{[i]}$ is a $\mathbb{Z}$-graded Lie superalgebra, where

$$
K_{[i]}=\operatorname{span}_{\mathbb{F}}\left\{x^{(\alpha)} x^{u}|| \alpha\left|+\alpha_{m}+\right| u \mid=i+2\right\} .
$$

Let $K_{j}=\bigoplus_{i \geq j} K_{[i]}$ for $j \geq-2$. Then $K=K_{-2} \supset K_{-1} \supset K_{0} \supset$ $\cdots$ is referred to as the natural filtration of $K$.

Lemma 4. $K=\bigoplus_{i=-2}^{\infty} K_{[i]}$ is transitively graded.

Proof. Assume the contrary, then there exists $y \in K_{[\ell]}$ such that $\llbracket y, x_{j} \rrbracket=0$ for all $j \in J$, where $\ell \geq 0$. Suppose that the largest exponent of $x_{m}$ among the nonzero summands in the expression of $y$ is equal to $t$, and write

$$
y=\sum_{\alpha, u, \alpha_{m}=t} c_{\alpha, u} x^{(\alpha)} x^{u}+\sum_{\beta, v, \beta_{m}<t} d_{\beta, v} x^{(\beta)} x^{v},
$$


where $c_{\alpha, u}, d_{\beta, v} \in \mathbb{F}$. Hence, for $j \in J_{0}$,

$$
0=\llbracket y, x_{j} \rrbracket=\sum_{\alpha, u, \alpha_{m}=t} \sigma\left(j^{\prime}\right) c_{\alpha, u} x^{\left(\alpha-\varepsilon_{j}\right)} x^{u}+h,
$$

where each exponent of $x_{m}$ of all nonzero summands in the expression of $h$ is less than $t$. Then $\sum_{\alpha, u, \alpha_{m}=t} \sigma\left(j^{\prime}\right) c_{\alpha, u} x^{\left(\alpha-\varepsilon_{j}\right)} x^{u}=0$. Note that all nonzero summands of $\sum_{\alpha, u, \alpha_{m}=t} \sigma\left(j^{\prime}\right) c_{\alpha, u} x^{\left(\alpha-\varepsilon_{j}\right)} x^{u}$ are $\mathbb{F}$-linear independent. It follows that each $x^{\left(\alpha-\varepsilon_{j}\right)} x^{u}$ is equal to 0 . Hence the exponents of $x_{j}$ in each $x^{(\alpha)} x^{u}$ are equal to 0 . Similarly, for $j \in Y_{1}$, we can prove that $x_{j}$ does not appear in each $x^{(\alpha)} x^{u}$. Consequently, we see that all $x^{(\alpha)} x^{u}$ are of the form $x^{\left(t \varepsilon_{m}\right)}$. If $t=0$, then $y \in K_{[-2]}$, contradicting $\ell \geq 0$. Hence $t>0$, and we can write

$$
y=c_{t \varepsilon_{m}} x^{\left(t \varepsilon_{m}\right)}+\sum_{\alpha, u, \alpha_{m}=t-1} c_{\alpha, u} x^{(\alpha)} x^{u}+\sum_{\beta, v, \beta_{m}<t-1} d_{\beta, v} x^{(\beta)} x^{v},
$$

where $c_{t \varepsilon_{m}} \neq 0$. Note that, for $j \in Y_{1}$,

$$
\begin{aligned}
0= & \llbracket y, x_{j} \rrbracket=-c_{t \varepsilon_{m}} x^{\left((t-1) \varepsilon_{m}\right)} x_{j} \\
& +\sum_{\alpha, u, \alpha_{m}=t-1}(-1)^{|u|} c_{\alpha, u} x^{(\alpha)} D_{j}\left(x^{u}\right)+h,
\end{aligned}
$$

where each exponent of $x_{m}$ of the nonzero summands in the expression of $h$ is less than $t-1$. Therefore,

$$
-c_{t \varepsilon_{m}} x^{\left((t-1) \varepsilon_{m}\right)} x_{j}+\sum_{\alpha, u, \alpha_{m}=t-1}(-1)^{|u|} c_{\alpha, u} x^{(\alpha)} D_{j}\left(x^{u}\right)=0 .
$$

Since $x_{j}$ appears in $x^{\left((t-1) \varepsilon_{m}\right)} x_{j}$ and does not appear in $\sum_{\alpha, u, \alpha_{m}=t-1}(-1)^{|u|} c_{\alpha, u} x^{(\alpha)} D_{j}\left(x^{u}\right)$, we conclude that $t=0$, a contradiction.

\section{3. ad-Nilpotent Elements}

Recall that $y \in K$ is called ad-nilpotent if there exists $t \in \mathbb{N}$ such that $(\operatorname{ad} y)^{t}(K)=0$. For a subset $R$ of $K$, let nil $(R)$ denote the set of ad-nilpotent elements in $R$, and let $\mathrm{Nil}(R)$ denote the subalgebra of $K$ generated by $\operatorname{nil}(R)$.

Lemma 5. Suppose that $y_{[i]} \in K_{[i]}$ for $i \geq-2$. The following statements hold.

(1) If $y=\sum_{i=k}^{t} y_{[i]} \in \operatorname{nil}(K)$, then $y_{[k]} \in \operatorname{nil}(K)$.

(2) If $y=\sum_{i=-2}^{t} y_{[i]} \in \operatorname{nil}(K)$, then $y_{[-2]}=0$.

(3) If $y=\sum_{i=-2}^{t} y_{[i]} \in \operatorname{nil}\left(K_{\overline{0}}\right)$, then $y_{[-1]}=0$.

(4) If $y=\sum_{i=-2}^{t} y_{[i]} \in \operatorname{nil}\left(K_{\overline{0}}\right)$, then $y_{[0]} \in \operatorname{nil}\left(K_{\overline{0}}\right)$.

(5) If $y=\sum_{i=-2}^{t} y_{[i]} \in \operatorname{nil}(K)$, then $y_{[-1]} \in \operatorname{span}_{\mathbb{F}}\left\{x_{j} \mid j \in\right.$ $\left.Y_{1}\right\}$.

Proof. (1) See [15, Lemma 5.1].
(2) Suppose that $y_{[-2]} \neq 0$. As $y$ is ad-nilpotent, $y_{[-2]}$ is adnilpotent by (1). Note that

$$
(\operatorname{ad} 1)^{k}\left(x^{\left(k \varepsilon_{m}\right)}\right)=(\operatorname{ad} 1)^{k-1}\left(2 x^{\left((k-1) \varepsilon_{m}\right)}\right)=2^{k} \neq 0,
$$

for all $k>0$. This shows that $y_{[-2]}$ is not ad-nilpotent, a contradiction.

(3) By (1), we see that $y_{[-1]}$ is ad-nilpotent. Suppose that $y_{[-1]}=\sum_{i \in J_{0}} a_{i} x_{i} \neq 0$, where $a_{i} \in \mathbb{F}$. Then there exists some $a_{j} \neq 0$. A direct calculation shows that

$$
\begin{aligned}
\left(\operatorname{ad} y_{[-1]}\right)^{k}\left(x^{\left(k \varepsilon_{j^{\prime}}\right)}\right) & =\left(\operatorname{ad} y_{[-1]}\right)^{k-1}\left(\sigma(j) a_{j} x^{\left((k-1) \varepsilon_{j^{\prime}}\right)}\right) \\
& =\sigma(j)^{k} a_{j}^{k} \neq 0,
\end{aligned}
$$

for all $k>0$. It follows that $y_{[-1]}$ is not ad-nilpotent.

(4) is an immediate consequence of (2), (3), and (1). (5) follows from (2), (1), and the proof of (3).

Let $a \in \mathbb{N}_{0}$ and $a=\sum_{l=0}^{\infty} a_{l} p^{l}$ be the $p$-adic expression of $a$, where $0 \leq a_{l}<p$. Then,

$$
\operatorname{pad}(a)=\left(\operatorname{pad}_{0}(a), \operatorname{pad}_{1}(a), \operatorname{pad}_{2}(a), \ldots\right)
$$

is said to be the $p$-adic sequence of $a$, where $\operatorname{pad}_{j}(a)=a_{j}$ for all $j \in \mathbb{N}_{0}$. For $\alpha=\left(\alpha_{1}, \alpha_{2}, \ldots, \alpha_{m}\right) \in \mathbb{N}_{0}^{m}$, define the $p$-adic matrix of $\alpha$ to be

$$
\operatorname{pad}(\alpha)=\left(\begin{array}{c}
\operatorname{pad}\left(\alpha_{1}\right) \\
\operatorname{pad}\left(\alpha_{2}\right) \\
\vdots \\
\operatorname{pad}\left(\alpha_{m}\right)
\end{array}\right)
$$

As $\operatorname{pad}(\alpha)$ is an $m \times \infty$ matrix with finitely many nonzero elements,

$$
\text { ht }(\alpha)=\max \left\{j \in \mathbb{N}_{0} \mid \exists i \in Y_{0}: \operatorname{pad}_{j}\left(\alpha_{i}\right) \neq 0\right\}
$$

is well defined. Let

$$
\|\alpha\|_{b, c}=\sum_{i=1}^{m} \sum_{j=b}^{c} \operatorname{pad}_{j}\left(\alpha_{i}\right),
$$

for $b \in \mathbb{N}_{0}$ and $c \in \mathbb{N}$. We abbreviate $\|\alpha\|_{0, q}$ to $\|\alpha\|_{q}$.

Suppose that $y=\sum_{\alpha, u} c_{\alpha, u} x^{(\alpha)} x^{u}$ is a nonzero element of $K$, where $c_{\alpha, u} \in \mathbb{F}$. Define

$$
\text { ht }(y)=\max \left\{\text { ht }(\alpha) \mid c_{\alpha, u} \neq 0\right\} \text {. }
$$

Given that $q>0$ and $x^{(\alpha)} x^{u} \in K$, we define

$$
\mathfrak{F}_{q}\left(x^{(\alpha)} x^{u}\right)=\|\alpha\|_{q}+2\|\alpha\|_{1, q}+|u|+\operatorname{pad}_{0}\left(\alpha_{m}\right) \text {. }
$$

Lemma 6. Let $\alpha, \beta \in \mathbb{N}_{0}^{m}, i \in Y_{0}$, and $q \in \mathbb{N}$. Then,

(1) $x^{(\alpha)} x^{(\beta)} \neq 0$ if and only if $\operatorname{pad}(\alpha)+\operatorname{pad}(\beta)=\operatorname{pad}(\alpha+\beta)$;

(2) If $\beta_{i} \neq 0$, then $\left\|\beta-\varepsilon_{i}\right\|_{q}+2\left\|\beta-\varepsilon_{i}\right\|_{1, q} \geq\|\beta\|_{q}+2\|\beta\|_{1, q}-$ 1 ; 
(3) If $x^{(\beta)} x^{v} \in K_{1}$ and $q \geq \operatorname{ht}\left(x^{(\beta)} x^{v}\right)$, then $\mathfrak{F}_{q}\left(x^{(\beta)} x^{v}\right) \geq$ 3.

Proof. (1) See [7, Lemma 2.5].

(2) First consider the case $\operatorname{pad}_{0}\left(\beta_{i}\right) \neq 0$. Then,

$$
\operatorname{pad}\left(\beta_{i}-1\right)=\left(\operatorname{pad}_{0}\left(\beta_{i}\right)-1, \operatorname{pad}_{1}\left(\beta_{i}\right), \ldots\right) .
$$

It follows that $\left\|\beta-\varepsilon_{i}\right\|_{q}=\|\beta\|_{q}-1$ and $\left\|\beta-\varepsilon_{i}\right\|_{1, q}=\|\beta\|_{1, q}$, and thus (2) holds.

Next consider the case $\operatorname{pad}_{0}\left(\beta_{i}\right)=0$. We may assume that

$$
\begin{aligned}
\operatorname{pad} & \left(\beta_{i}\right) \\
& =\left(0, \ldots, 0, \operatorname{pad}_{t}\left(\beta_{i}\right), \operatorname{pad}_{t+1}\left(\beta_{i}\right), \ldots\right),
\end{aligned}
$$

where $\operatorname{pad}_{t}\left(\beta_{i}\right) \neq 0$ and $t \geq 1$. Hence,

$$
\begin{aligned}
\operatorname{pad} & \left(\beta_{i}-1\right) \\
& =\left(p-1, \ldots, p-1, \operatorname{pad}_{t}\left(\beta_{i}\right)-1, \operatorname{pad}_{t+1}\left(\beta_{i}\right), \ldots\right) .
\end{aligned}
$$

If $q<t$, then

$$
\begin{aligned}
\left\|\beta-\varepsilon_{i}\right\|_{q}+2\left\|\beta-\varepsilon_{i}\right\|_{1, q} \\
=(q+1)(p-1)+2 q(p-1) \\
>-1 \\
=\|\beta\|_{q}+2\|\beta\|_{1, q}-1 .
\end{aligned}
$$

If $q=t$, noting that $p>2$, then

$$
\begin{aligned}
\| \beta & -\varepsilon_{i}\left\|_{q}+2\right\| \beta-\varepsilon_{i} \|_{1, q} \\
& =t(p-1)+2(t-1)(p-1)+3\left(\operatorname{pad}_{t}\left(\beta_{i}\right)-1\right) \\
& \geq 2+3 \operatorname{pad}_{t}\left(\beta_{i}\right)-3 \\
& =3 \operatorname{pad}_{t}\left(\beta_{i}\right)-1 \\
& =\|\beta\|_{q}+2\|\beta\|_{1, q}-1 .
\end{aligned}
$$

If $q>t$, then

$$
\begin{aligned}
\| \beta & -\varepsilon_{i}\left\|_{q}+2\right\| \beta-\varepsilon_{i} \|_{1, q} \\
& =\left\|\beta-\varepsilon_{i}\right\|_{t}+2\left\|\beta-\varepsilon_{i}\right\|_{1, t}+3\left\|\beta-\varepsilon_{i}\right\|_{t+1, q} \\
& \geq\|\beta\|_{t}+2\|\beta\|_{1, t}-1+3\left\|\beta-\varepsilon_{i}\right\|_{t+1, q} \\
& =\|\beta\|_{q}+2\|\beta\|_{1, q}-1 .
\end{aligned}
$$

(3) The assumption $x^{(\beta)} x^{v} \in K_{1}$ implies that $|\beta|+\beta_{m}+|v| \geq$ 3. Then it is trivially verified that (3) holds.

Lemma 7. Suppose that $x^{(\beta)} x^{v} \in K_{1}, x^{(\alpha)} x^{u} \in K, q \geq$ $\max \left\{1, \operatorname{ht}\left(x^{(\beta)} x^{v}\right)\right\}$, and $i \in J$. The following statements hold.

(1) If $x^{(\beta)} x^{v} D_{m}\left(x^{(\alpha)} x^{u}\right) \neq 0$, then $\mathfrak{F}_{q}\left(x^{(\beta)} x^{v} D_{m}\left(x^{(\alpha)} x^{u}\right)\right) \geq$ $\mathfrak{F}_{q}\left(x^{(\alpha)} x^{u}\right)+1$.
(2) If $x^{(\alpha)} x^{u} D_{m}\left(x^{(\beta)} x^{v}\right) \neq 0$, then $\mathfrak{F}_{q}\left(x^{(\alpha)} x^{u} D_{m}\left(x^{(\beta)} x^{v}\right)\right) \geq$ $\mathfrak{\mho}_{q}\left(x^{(\alpha)} x^{u}\right)+1$.

(3) If $D_{i}\left(x^{(\beta)} x^{v}\right) D_{i^{\prime}}\left(x^{(\alpha)} x^{u}\right) \neq 0$, then $\mathfrak{F}_{q}\left(D_{i}\left(x^{(\beta)} x^{v}\right)\right.$ $\left.D_{i^{\prime}}\left(x^{(\alpha)} x^{u}\right)\right) \geq \mathfrak{\mho}_{q}\left(x^{(\alpha)} x^{u}\right)+1$.

Proof. (1) The assumption $x^{(\beta)} x^{v} D_{m}\left(x^{(\alpha)} x^{u}\right) \neq 0$ implies that $x^{(\beta)} x^{\left(\alpha-\varepsilon_{m}\right)} \neq 0$. By Lemma 6(1), we have

$$
\operatorname{pad}\left(\beta+\left(\alpha-\varepsilon_{m}\right)\right)=\operatorname{pad}(\beta)+\operatorname{pad}\left(\alpha-\varepsilon_{m}\right) .
$$

Consequently,

$$
\begin{gathered}
\operatorname{pad}_{0}\left(\beta_{m}+\left(\alpha_{m}-1\right)\right)=\operatorname{pad}_{0}\left(\beta_{m}\right)+\operatorname{pad}_{0}\left(\alpha_{m}-1\right), \\
\left\|\beta+\left(\alpha-\varepsilon_{m}\right)\right\|_{q}=\|\beta\|_{q}+\left\|\alpha-\varepsilon_{m}\right\|_{q} \\
\left\|\beta+\left(\alpha-\varepsilon_{m}\right)\right\|_{1, q}=\|\beta\|_{1, q}+\left\|\alpha-\varepsilon_{m}\right\|_{1, q^{*}}
\end{gathered}
$$

By (2) and (3) of Lemma 6, we obtain

$$
\begin{gathered}
\left\|\alpha-\varepsilon_{m}\right\|_{q}+\left\|\alpha-\varepsilon_{m}\right\|_{1, q} \geq\|\alpha\|_{q}+\|\alpha\|_{1, q}-1, \\
\mathfrak{\mho}_{q}\left(x^{(\beta)} x^{v}\right) \geq 3 .
\end{gathered}
$$

Combining (34)-(38), we have

$$
\begin{aligned}
\mathfrak{F}_{q}( & \left.x^{(\beta)} x^{v} D_{m}\left(x^{(\alpha)} x^{u}\right)\right) \\
= & \mathfrak{F}_{q}\left(x^{\left(\beta+\left(\alpha-\varepsilon_{m}\right)\right)} x^{v} x^{u}\right) \\
= & \left\|\beta+\left(\alpha-\varepsilon_{m}\right)\right\|_{q}+2\left\|\beta+\left(\alpha-\varepsilon_{m}\right)\right\|_{1, q}+|u|+|v| \\
& +\operatorname{pad}_{0}\left(\beta_{m}+\left(\alpha_{m}-1\right)\right) \\
= & \|\beta\|_{q}+\left\|\alpha-\varepsilon_{m}\right\|_{q}+2\|\beta\|_{1, q}+2\left\|\alpha-\varepsilon_{m}\right\|_{1, q}+|u|+|v| \\
& +\operatorname{pad}_{0}\left(\beta_{m}\right)+\operatorname{pad}_{0}\left(\alpha_{m}-1\right) \\
= & \|\beta\|_{q}+2\|\beta\|_{1, q}+|v|+\operatorname{pad}_{0}\left(\beta_{m}\right)+\left\|\alpha-\varepsilon_{m}\right\|_{q} \\
& +2\left\|\alpha-\varepsilon_{m}\right\|_{1, q}+|u|+\operatorname{pad}_{0}\left(\alpha_{m}-1\right) \\
\geq & \mathfrak{F}_{q}\left(x^{(\beta)} x^{v}\right)+\|\alpha\|_{q}+2\|\alpha\|_{1, q}-1+|u|+\operatorname{pad}_{0}\left(\alpha_{m}\right)-1 \\
\geq & \mathfrak{F}_{q}\left(x^{(\alpha)} x^{u}\right)+1 .
\end{aligned}
$$

(2) Suppose that $x^{(\alpha)} x^{u} D_{m}\left(x^{(\beta)} x^{v}\right) \neq 0$. Then,

$$
\begin{aligned}
\mathfrak{F}_{q} & \left(x^{(\alpha)} x^{u} D_{m}\left(x^{(\beta)} x^{v}\right)\right) \\
= & \mathfrak{F}_{q}\left(x^{\left(\alpha+\left(\beta-\varepsilon_{m}\right)\right)} x^{v} x^{u}\right) \\
= & \left\|\alpha+\left(\beta-\varepsilon_{m}\right)\right\|_{q}+2\left\|\alpha+\left(\beta-\varepsilon_{m}\right)\right\|_{1, q}+|u|+|v| \\
& +\operatorname{pad}_{0}\left(\alpha_{m}+\left(\beta_{m}-1\right)\right)
\end{aligned}
$$




$$
\begin{aligned}
= & \|\alpha\|_{q}+\left\|\beta-\varepsilon_{m}\right\|_{q}+2\|\alpha\|_{1, q}+2\left\|\beta-\varepsilon_{m}\right\|_{1, q}+|u|+|v| \\
& +\operatorname{pad}_{0}\left(\alpha_{m}\right)+\operatorname{pad}_{0}\left(\beta_{m}-1\right) \\
= & \|\alpha\|_{q}+2\|\alpha\|_{1, q}+|u|+\operatorname{pad}_{0}\left(\alpha_{m}\right)+\left\|\beta-\varepsilon_{m}\right\|_{q} \\
& +2\left\|\beta-\varepsilon_{m}\right\|_{1, q}+|v|+\operatorname{pad}_{0}\left(\beta_{m}-1\right) \\
\geq & \mathfrak{\mho}_{q}\left(x^{(\alpha)} x^{u}\right)+\|\beta\|_{q}+2\|\beta\|_{1, q}-1+|v|+\operatorname{pad}_{0}\left(\beta_{m}\right)-1 \\
\geq & \mathfrak{\mho}_{q}\left(x^{(\alpha)} x^{u}\right)+1 .
\end{aligned}
$$

(3) Similarly, we have

$$
\begin{aligned}
\mathfrak{F}_{q}( & \left.D_{i}\left(x^{(\beta)} x^{v}\right) D_{i^{\prime}}\left(x^{(\alpha)} x^{u}\right)\right) \\
= & \mathfrak{\mho}_{q}\left(x^{\left(\left(\beta-\varepsilon_{i}\right)+\left(\alpha-\varepsilon_{i^{\prime}}\right)\right)} x^{v} x^{u}\right) \\
= & \left\|\left(\beta-\varepsilon_{i}\right)+\left(\alpha-\varepsilon_{i^{\prime}}\right)\right\|_{q}+2\left\|\left(\beta-\varepsilon_{i}\right)+\left(\alpha-\varepsilon_{i^{\prime}}\right)\right\|_{1, q}+|v| \\
& +|u|+\operatorname{pad}_{0}\left(\beta_{m}+\alpha_{m}\right) \\
= & \left\|\beta-\varepsilon_{i}\right\|_{q}+\left\|\alpha-\varepsilon_{i^{\prime}}\right\|_{q}+2\left\|\beta-\varepsilon_{i}\right\|_{1, q}+2\left\|\alpha-\varepsilon_{i^{\prime}}\right\|_{1, q}+|v| \\
& +|u|+\operatorname{pad}_{0}\left(\beta_{m}\right)+\operatorname{pad}_{0}\left(\alpha_{m}\right) \\
= & \left\|\beta-\varepsilon_{i}\right\|_{q}+2\left\|\beta-\varepsilon_{i}\right\|_{1, q}+\left\|\alpha-\varepsilon_{i^{\prime}}\right\|_{q}+2\left\|\alpha-\varepsilon_{i^{\prime}}\right\|_{1, q}+|v| \\
& +|u|+\operatorname{pad}_{0}\left(\beta_{m}\right)+\operatorname{pad}_{0}\left(\alpha_{m}\right) \\
\geq & \|\beta\|_{q}+2\|\beta\|_{1, q}-1+\|\alpha\|_{q}+2\|\alpha\|_{1, q}-1+|v|+|u| \\
& +\operatorname{pad}_{0}\left(\beta_{m}\right)+\operatorname{pad}_{0}\left(\alpha_{m}\right) \\
= & \mathfrak{\mho}_{q}\left(x^{(\beta)} x^{v}\right)+\mathfrak{\mho}_{q}\left(x^{(\alpha)} x^{u}\right)-2 \\
\geq & \mathfrak{\mho}_{q}\left(x^{(\alpha)} x^{u}\right)+1 .
\end{aligned}
$$

Lemma 8. Suppose that $x^{(\beta)} x^{v} \in K_{1}, x^{(\alpha)} x^{u} \in K$, and $q \geq \max \left\{1, \mathrm{ht}\left(x^{(\beta)} x^{\nu}\right)\right\}$. Let $x^{\left(\alpha^{\prime}\right)} x^{u^{\prime}}$ be a nonzero summand of $\llbracket x^{(\beta)} x^{v}, x^{(\alpha)} x^{u} \rrbracket$. Then $\mathfrak{F}_{q}\left(x^{\left(\alpha^{\prime}\right)} x^{u^{\prime}}\right) \geq \mathfrak{F}_{q}\left(x^{(\alpha)} x^{u}\right)+1$.

Proof. A direct calculation shows that $x^{\left(\alpha^{\prime}\right)} x^{u^{\prime}}$ fulfills the conditions of Lemma 7.

\section{Given that $q \in \mathbb{N}$, let}

$$
\ell_{q}=m(q+1)(p-1)+2 m q(p-1)+(p-1)+n+1 .
$$

Clearly, the inequality $\mathfrak{F}_{q}\left(x^{(\alpha)} x^{u}\right)<\ell_{q}$ holds for all $x^{(\alpha)} x^{u} \in$ $K$.

Lemma 9. $K_{1} \subset \operatorname{nil}(K)$.
Proof. Suppose that $y=\sum_{\beta, u} c_{\alpha, v} x^{(\beta)} x^{v}$ is an arbitrary element of $K_{1}$, where $c_{\beta, v} \in \mathbb{F}$ and $c_{\beta, v} \neq 0$. Let $q \in \mathbb{N}$ such that $q \geq \operatorname{ht}(y)$. Let $x^{(\alpha)} x^{u}$ be a standard basis element of $K$. By using Lemma 8 repeatedly, we see that $(\operatorname{ad} y)^{\ell_{q}}\left(x^{(\alpha)} x^{u}\right)=$ 0.

Lemma 10. For $i, j \in J_{0}$, the following statements hold.

(1) $x^{\left(2 \varepsilon_{i}\right)} \in \operatorname{nil}\left(K_{[0]} \cap K_{\overline{0}}\right)$.

(2) If $\sigma(i)=\sigma(j)$ and $i \neq j$, then $x_{i} x_{j} \in \operatorname{nil}\left(K_{[0]} \cap K_{\overline{0}}\right)$.

(3) If $\sigma(i) \neq \sigma(j)$ and $i \neq j^{\prime}$, then $x_{i} x_{j} \in \operatorname{nil}\left(K_{[0]} \cap K_{\overline{0}}\right)$.

(4) $x_{i} x_{i^{\prime}} \in \operatorname{Nil}\left(K_{[0]} \cap K_{\overline{0}}\right)$.

Proof. (1) Let $x^{(\alpha)} x^{u}$ be a standard basis element of K. A direct calculation shows that

$$
\begin{aligned}
\left(\operatorname{ad} x^{\left(2 \varepsilon_{i}\right)}\right)^{p}\left(x^{(\alpha)} x^{u}\right) & =\left(\operatorname{ad} x^{\left(2 \varepsilon_{i}\right)}\right)^{p-1}\left(\sigma(i) x_{i} x^{\left(\alpha-\varepsilon_{i^{\prime}}\right)} x^{u}\right) \\
& =\sigma(i)^{p} x_{i}^{p} x^{\left(\alpha-p \varepsilon_{i}^{\prime}\right)} x^{u}=0 .
\end{aligned}
$$

(2) Since $\operatorname{ad} x_{i} x_{j}=\sigma(i)\left(x_{j} D_{i^{\prime}}+x_{i} D_{j^{\prime}}\right)$ and $x_{j} D_{i^{\prime}}$ 。 $x_{i} D_{j^{\prime}}=x_{i} D_{j^{\prime}} \circ x_{j} D_{i^{\prime}}$, it follows from the binomial theorem that $\left(\operatorname{ad} x_{i} x_{j}\right)^{p}=\sigma(i)^{p}\left(x_{j} D_{i^{\prime}}+x_{i} D_{j^{\prime}}\right)^{p}=\sigma(i)^{p}\left(\left(x_{j} D_{i^{\prime}}\right)^{p}+\right.$ $\left.\left(x_{i} D_{j^{\prime}}\right)^{p}\right)=0$.

(3) Since ad $x_{i} x_{j}=\sigma(i)\left(x_{j} D_{i^{\prime}}-x_{i} D_{j^{\prime}}\right)$ and $x_{j} D_{i^{\prime}} \circ x_{i} D_{j^{\prime}}=$ $x_{i} D_{j^{\prime}} \circ x_{j} D_{i^{\prime}}$, we have $\left(\operatorname{ad} x_{i} x_{j}\right)^{p}=\sigma(i)^{p}\left(x_{j} D_{i^{\prime}}-x_{i} D_{j^{\prime}}\right)^{p}=$ $\sigma(i)^{p}\left(\left(x_{j} D_{i^{\prime}}\right)^{p}-\left(x_{i} D_{j^{\prime}}\right)^{p}\right)=0$.

(4) It follows from (1) that $x_{i} x_{i^{\prime}}=\sigma(i) \llbracket x^{\left(2 \varepsilon_{i}\right)}, x^{\left(2 \varepsilon_{i^{\prime}}\right)} \rrbracket \in$ $\operatorname{Nil}\left(K_{[0]} \cap K_{\overline{0}}\right)$.

Lemma 11. Suppose that $n \geq 3$. The following statements hold.

(1) Let $i, j, k$ be distinct elements of $Y_{1}$, and let $a, b \in \mathbb{F}$ be such that $a^{2}+b^{2}=0$. Then $y=a x_{i} x_{j}+b x_{i} x_{k} \in \operatorname{nil}(K)$.

(2) $x_{i} x_{j} \in \operatorname{Nil}\left(K_{[0]} \cap K_{\overline{0}}\right)$ holds for all distinct $i, j \in Y_{1}$.

Proof. (1) A direct calculation shows that $\operatorname{ad} y=$ $a x_{j} D_{i}-a x_{i} D_{j}+b x_{k} D_{i}-b x_{i} D_{k}$. For simplicity, we denote $a x_{j} D_{i},-a x_{i} D_{j}, b x_{k} D_{i},-b x_{i} D_{k}$ by $A, B, C, D$, respectively. Clearly,

$$
A^{2}=B^{2}=C^{2}=D^{2}=0, \quad A C=C A=B D=D B=0 .
$$

Then

$$
(\operatorname{ad} y)^{2}=A B+B A+A D+D A+B C+C B+D C+C D .
$$

By $A D A=D A D=B C B=C B C=0$, we obtain

$$
\begin{aligned}
(\operatorname{ad} y)^{3}= & A B A+A B C+A D C+B A B+B A D+B C D \\
& +C B A+C D A+C D C+D A B+D C B+D C D .
\end{aligned}
$$


Note that

$$
\begin{gathered}
A B A=-a^{2} A, \quad B A B=-a^{2} B, \\
C D C=-b^{2} C, \quad D C D=-b^{2} D, \\
a^{2} A=C D A+A D C, \quad a^{2} B=B C D+D C B, \\
b^{2} C=A B C+C B A, \quad b^{2} D=B A D+D A B .
\end{gathered}
$$

It follows that

$$
\begin{array}{ll}
A B A+C D A+A D C=0, & B A B+B C D+D C B=0, \\
C D C+A B C+C B A=0, & D C D+B A C+D A B=0,
\end{array}
$$

thus proving that $(\operatorname{ad} y)^{3}=0$.

(2) Let $a \in \mathbb{F}$ such that $a^{2}=-1$. Since $\operatorname{char}(\mathbb{F})>2$, we have $a^{2}-1=-2 \neq 0$. Let $k \in Y_{1} \backslash\{i, j\}$. Then (1) yields

$$
\begin{aligned}
& y_{1}=a x_{i} x_{j}+x_{i} x_{k} \in \operatorname{Nil}\left(H_{[0]} \cap H_{\overline{0}}\right), \\
& y_{2}=x_{i} x_{j}+a x_{i} x_{k} \in \operatorname{Nil}\left(H_{[0]} \cap H_{\overline{0}}\right) .
\end{aligned}
$$

Hence $x_{i} x_{j}=-(1 / 2)\left(a y_{1}-y_{2}\right) \in \operatorname{Nil}\left(H_{[0]} \cap H_{\overline{0}}\right)$.

Lemma 12. Let $i \in J_{0}$ and $j \in Y_{1}$. Then $x_{i} x_{j} \in \operatorname{nil}\left(K_{[0]} \cap K_{\overline{1}}\right)$.

Proof. A direct calculation shows that

$$
\begin{gathered}
\operatorname{ad} x_{i} x_{j}=\sigma(i) x_{j} D_{i^{\prime}}-x_{i} D_{j}, \\
\left(\operatorname{ad} x_{i} x_{j}\right)^{2}=-\sigma(i)\left(x_{i} D_{j} \circ x_{j} D_{i^{\prime}}+x_{j} D_{i^{\prime}} \circ x_{i} D_{j}\right) .
\end{gathered}
$$

Since

$$
\begin{aligned}
& \left(x_{i} D_{j} \circ x_{j} D_{i^{\prime}}\right) \circ\left(x_{j} D_{i^{\prime}} \circ x_{i} D_{j}\right) \\
& =0=\left(x_{j} D_{i^{\prime}} \circ x_{i} D_{j}\right) \circ\left(x_{i} D_{j} \circ x_{j} D_{i^{\prime}}\right),
\end{aligned}
$$

we have

$$
\begin{aligned}
& \left(\operatorname{ad} x_{i} x_{j}\right)^{2 p} \\
& \quad=-\sigma(i)\left(\left(x_{i} D_{j} \circ x_{j} D_{i^{\prime}}\right)^{p}+\left(x_{j} D_{i^{\prime}} \circ x_{i} D_{j}\right)^{p}\right)=0 .
\end{aligned}
$$

Lemma 13. (1) $\mathrm{Nil}\left(K_{[0]}\right)=\operatorname{span}_{\mathbb{F}}\left\{x_{i} x_{j} \mid i, j \in J\right\}$.

(2) $\operatorname{Nil}\left(K_{0}\right)=\operatorname{Nil}\left(K_{[0]}\right)+K_{1}$.

(3) If $n \geq 3$, then $\operatorname{Nil}\left(K_{[0]} \cap K_{\overline{0}}\right)=\operatorname{span}_{\mathbb{F}}\left\{x_{i} x_{j} \mid i, j \in\right.$ $J, \mu(i)=\mu(j)\}$.

(4) If $n \leq 2$, then $\operatorname{Nil}\left(K_{[0]} \cap K_{\overline{0}}\right)=\operatorname{span}_{\mathbb{E}}\left\{x_{i} x_{j} \mid i, j \in J_{0}\right\}$.

(5) $\operatorname{Nil}\left(K_{0} \cap K_{\overline{0}}\right)=\operatorname{Nil}\left(K_{[0]} \cap K_{\overline{0}}\right)+K_{1} \cap K_{\overline{0}}$.

Proof. (1) Let $y=a_{m} x_{m}+\sum_{i, j \in J} a_{i j} x_{i} x_{j}$ be an arbitrary element of $\operatorname{nil}\left(K_{[0]}\right)$. Suppose that $a_{m} \neq 0$. Since $(\operatorname{ad} y)^{t}(1)=$ $\left(-2 a_{m}\right)^{t} \neq 0$ and $\forall t \in \mathbb{N}$, it follows that $y$ is not ad-nilpotent, a contradiction. Hence $a_{m}=0$, and therefore $\operatorname{nil}\left(K_{[0]}\right) \subseteq$ $\operatorname{span}_{\mathbb{F}}\left\{x_{i} x_{j} \mid i, j \in J\right\}$. Noting that $\operatorname{span}_{\mathbb{F}}\left\{x_{i} x_{j} \mid i, j \in J\right\}$ is a subalgebra of $K$, we obtain $\operatorname{Nil}\left(K_{[0]}\right) \subseteq \operatorname{span}_{\mathbb{F}}\left\{x_{i} x_{j} \mid i, j \in J\right\}$.
Conversely, Lemma 10 shows that $\operatorname{span}_{\mathbb{F}}\left\{x_{i} x_{j} \mid i, j \in J_{0}\right\} \subseteq$ $\operatorname{Nil}\left(K_{[0]}\right)$, and Lemma 12 implies that $\operatorname{span}_{\mathbb{F}}\left\{x_{i} x_{j} \mid i \in J_{0}, j \in\right.$ $\left.Y_{1}\right\} \subseteq \operatorname{Nil}\left(K_{[0]}\right)$. Moreover, since $x_{i} x_{j}=\llbracket x_{1} x_{i}, x_{1}, x_{j} \rrbracket$ for all $i, j \in Y_{1}$, we have $\operatorname{span}_{\mathbb{F}}\left\{x_{i} x_{j} \mid i, j \in Y_{1}\right\} \subseteq \operatorname{Nil}\left(K_{[0]}\right)$. Therefore $\operatorname{span}_{\mathbb{F}}\left\{x_{i} x_{j} \mid i, j \in J\right\} \subseteq \operatorname{Nil}\left(K_{[0]}\right)$.

(2) It is clear that $\operatorname{Nil}\left(K_{[0]}\right) \subseteq \operatorname{Nil}\left(K_{0}\right)$, which combined with Lemma 9 yields $\operatorname{Nil}\left(K_{[0]}\right)+K_{1} \subseteq \operatorname{Nil}\left(K_{0}\right)$.

On the other hand, suppose that $y=y_{[0]}+y_{1}$ is an arbitrary element of nil $\left(K_{0}\right)$, where $y_{[0]} \in K_{[0]}$ and $y_{1} \in K_{1}$. By Lemma 5, we have $y_{[0]} \in \operatorname{Nil}\left(K_{[0]}\right)$, and hence $y=y_{[0]}+$ $y_{1} \in \operatorname{Nil}\left(K_{[0]}\right)+K_{1}$. Since $\operatorname{Nil}\left(K_{[0]}\right)+K_{1}$ is a subalgebra of $K$, it follows that $\operatorname{Nil}\left(K_{0}\right) \subseteq \operatorname{Nil}\left(K_{[0]}\right)+K_{1}$.

(3) By (1), we see that $\operatorname{Nil}\left(K_{[0]} \cap K_{\overline{0}}\right) \subseteq \operatorname{Nil}\left(K_{[0]}\right) \cap K_{\overline{0}}=$ $\operatorname{span}_{\mathbb{F}}\left\{x_{i} x_{j} \mid i, j \in J, \mu(i)=\mu(j)\right\}$. The reverse inclusion follows from Lemmas 10 and 11 .

(4) Clearly the statement holds when $n=1$. Now we consider the case $n=2$. By (1), we can suppose that $y=$ $a x_{m+1} x_{s}+\sum_{i, j \in J_{0}} a_{i j} x_{i} x_{j}$ is an arbitrary element of $\operatorname{nil}\left(K_{[0]} \cap\right.$ $\left.K_{\overline{0}}\right)$, where $a, a_{i j} \in \mathbb{F}$. If $a \neq 0$, a direct calculation shows that $(\operatorname{ad} y)^{2 t}\left(x_{s}\right)=(-1)^{t} a^{2 t} x_{s} \neq 0$ for all $t \in \mathbb{N}$, contradicting that $y$ is ad-nilpotent. Hence $a=0$ and $y \in \operatorname{span}_{\mathbb{F}}\left\{x_{i} x_{j} \mid i, j \in J_{0}\right\}$, proving $\operatorname{nil}\left(K_{[0]} \cap K_{\overline{0}}\right) \subseteq \operatorname{span}_{\mathbb{F}}\left\{x_{i} x_{j} \mid i, j \in J_{0}\right\}$. Since $\operatorname{span}_{\mathbb{F}}\left\{x_{i} x_{j} \mid i, j \in J_{0}\right\}$ is a subalgebra of $K$, it follows that $\operatorname{Nil}\left(K_{[0]} \cap K_{\overline{0}}\right) \subseteq \operatorname{span}_{\mathbb{F}}\left\{x_{i} x_{j} \mid i, j \in J_{0}\right\}$. The reverse inclusion follows from Lemma 10.

(5) is completely analogous to the proof of (2).

Let $\rho$ be the corresponding representation with respect to $K_{[0]}$-module $K_{[-1]}$; that is, $\rho(y)=\left.\operatorname{ad} y\right|_{K_{[-1]}}, \forall y \in K_{[0]}$. It is easy to see that $\rho$ is faithful. For $y \in K_{[0]}$, we also denote by $\rho(y)$ the matrix of $\rho(y)$ relative to the fixed ordered $\mathbb{F}$-basis as follows:

$$
\left\{x_{1}, x_{2}, \ldots, x_{m-1}, x_{m+1}, \ldots, x_{s}\right\} .
$$

Denote by $\mathrm{gl}(2 r, n)$ the general linear Lie superalgebra of $(2 r+$ $n) \times(2 r+n)$ matrices over $\mathbb{F}$. Let $e_{i j}$ denote the $(s-1) \times(s-$ 1) matrix whose $(i, j)$-entry is 1 and 0 elsewhere, and $G=$ $\left(\begin{array}{cc}0 & I_{r} \\ -I_{r} & 0\end{array}\right)$, where $I_{r}$ is $r \times r$ unit matrix. Let $\operatorname{sp}(2 r, \mathbb{F})$ be the Lie algebra consisting of all $2 r \times 2 r$ matrices $A$ over $\mathbb{F}$ satisfying $A^{T} G+G A=0$, where $A^{T}$ is the transpose of $A$. Set $\mathscr{K}=$ $\mathscr{L} \oplus \mathbb{F} I_{s-1}$; here

$$
\mathscr{L}=\left\{\left(\begin{array}{ll}
A & B \\
C & D
\end{array}\right) \in \operatorname{gl}(2 r, n) \mid A \in \operatorname{sp}(2 r, \mathbb{F}), B^{T} G+C=0,\right.
$$

$$
D \text { anti-symmetric }\} \text {. }
$$

Lemma 14. (1) $\rho\left(K_{[0]}\right)=\mathscr{K}$.

(2) If $y \in \operatorname{nil}\left(K_{[0]}\right)$, then $\rho(y)$ is a nilpotent matrix.

Proof. (1) For $i, j \in J$, a direct calculation shows that

$$
\begin{aligned}
\llbracket x_{i} x_{j}, x_{k} \rrbracket= & \sigma(i)(-1)^{\mu(i)(\mu(i)+\mu(j))} \delta_{i^{\prime} k} x_{j} \\
& +\sigma(j)(-1)^{\mu(j)(\mu(i)+\mu(j))+\mu(i) \mu(j)} \delta_{j^{\prime} k} x_{i} .
\end{aligned}
$$


Therefore

$$
\rho\left(x_{i} x_{j}\right)=\sigma(i)(-1)^{\mu(i)+\mu(i) \mu(j)} e_{\widehat{\jmath i^{\prime}}}+\sigma(j)(-1)^{\mu(j)} e_{\widehat{i} j^{\prime}},
$$

where

$$
\widehat{l}= \begin{cases}l & \text { if } l \in J_{0} \\ l-1 & \text { if } l \in Y_{1} .\end{cases}
$$

Since $\llbracket x_{m}, x_{k} \rrbracket=-x_{k}, \forall k \in J$, we see that

$$
\rho\left(x_{m}\right)=-I_{s-1} .
$$

From (56) and (58), we can easily verify (1).

(2) follows from the definition of $\rho$.

If $A=\left(a_{i j}\right)$ is an $n \times n$ antisymmetric matrix over $\mathbb{F}$, we write $\Gamma(A)$ to denote $\sum_{1 \leq i<j \leq n} a_{i j}^{2}$. As $\operatorname{tr}\left(A^{2}\right)=-2 \sum_{1 \leq i<j \leq n} a_{i j}^{2}$, it is clear that if $A$ is a nilpotent matrix then $\Gamma(A)=0$.

Lemma 15. Suppose that $n \geq 3$. Let $A=\left(a_{i j}\right)$ be an antisymmetric matrix over $\mathbb{F}$ of order $n$. If $A$ satisfies the following properties:

(1) $\Gamma(A)=0$;

(2) $\Gamma([A, B])=0$ holds for every $n \times n$ antisymmetric matrix $B$.

Then, $A=0$.

Proof. Let $N=\{1,2, \ldots, n\}$. Three cases arise as follows.

Case $1(n>4)$. By the property (2) of $A$, we see that

$$
\begin{gathered}
\Gamma\left(\left[A, E_{i j}-E_{j i}+E_{k l}-E_{l k}\right]\right)-\Gamma\left(\left[A, E_{i j}-E_{j i}\right]\right) \\
-\Gamma\left(\left[A, E_{k l}-E_{l k}\right]\right)=0
\end{gathered}
$$

holds for all $i, j, k, l \in N$. Therefore, if $i<j<k<l$, a direct calculation shows that the left-hand side of (59) is equal to $4 a_{i l} a_{j k}-4 a_{i k} a_{j l}$. Hence $4 a_{i l} a_{j k}-4 a_{i k} a_{j l}=0$. Similarly, if $i<$ $k<j<l$, then $-4 a_{i l} a_{k j}-4 a_{i k} a_{j l}=0$, and if $i<k<l<j$, then $-4 a_{i l} a_{k j}+4 a_{i k} a_{l j}=0$. Thus, for all $i_{1}<i_{2}<i_{3}<i_{4}$, we see that $a_{i_{1} i_{3}} a_{i_{2} i_{4}}=a_{i_{1} i_{4}} a_{i_{2} i_{3}}=-a_{i_{1} i_{2}} a_{i_{3} i_{4}}=-a_{i_{1} i_{3}} a_{i_{2} i_{4}}$. Then

$$
a_{i_{1} i_{2}} a_{i_{3} i_{4}}=a_{i_{1} i_{3}} a_{i_{2} i_{4}}=a_{i_{1} i_{4}} a_{i_{2} i_{3}}=0 .
$$

Denote $\eta_{i}=\sum_{t=1}^{n} a_{i t}^{2}$ for $i \in N$. If $i \neq j$, a direct calculation shows that

$$
0=\Gamma\left(\left[A, E_{i j}-E_{j i}\right]\right)=\eta_{i}+\eta_{j}-2 a_{i j}^{2} .
$$

Let $k \in N \backslash\{i, j\}$. Then

$$
\eta_{i}+\eta_{k}-2 a_{i k}^{2}=0, \quad \eta_{j}+\eta_{k}-2 a_{j k}^{2}=0 .
$$

From the equalities above, we see that $\eta_{i}=a_{i j}^{2}+a_{i k}^{2}-a_{j k}^{2}$ holds for all distinct $i, j, k \in N$. Pick $l \in N \backslash\{i, j, k\}$. Then $\eta_{i}=a_{i j}^{2}+a_{i l}^{2}-a_{j l}^{2}$. Hence $a_{i l}^{2}+a_{j k}^{2}=a_{i k}^{2}+a_{j l}^{2}$ holds for all distinct $i, j, k, l \in N$. It follows that

$$
a_{i_{1} i_{2}}^{2}+a_{i_{3} i_{4}}^{2}=a_{i_{1} i_{3}}^{2}+a_{i_{2} i_{4}}^{2}=a_{i_{2} i_{3}}^{2}+a_{i_{1} i_{4}}^{2}
$$

holds for all $i_{1}<i_{2}<i_{3}<i_{4}$.
Assume that there exists some $a_{i j} \neq 0$. For distinct $k, l \in$ $N \backslash\{i, j\}$, we have $a_{k l}=0$ by (60). Then we can write the following:

$$
A=\sum_{t \in N} a_{i t} E_{i t}+\sum_{t \in N} a_{j t} E_{j t}-\sum_{t \in N} a_{i t} E_{t i}-\sum_{t \in N} a_{j t} E_{t j} .
$$

A direct calculation shows that

$$
0=\Gamma\left(\left[A, E_{k l}-E_{l k}\right]\right)=a_{i l}^{2}+a_{j l}^{2}+a_{i k}^{2}+a_{j k}^{2} .
$$

Since $a_{i l}^{2}+a_{j k}^{2}=a_{j l}^{2}+a_{i k}^{2}$ by (63), we obtain $a_{i l}^{2}+a_{j k}^{2}=0$. Hence $a_{i j}^{2}=a_{i j}^{2}+a_{k l}^{2}=a_{i l}^{2}+a_{j k}^{2}=0$ by (63), contradicting the assumption that $a_{i j} \neq 0$.

Case $2(n=4)$. Note that $(60)$ and $(63)$ hold; that is,

$$
\begin{aligned}
& a_{12} a_{34}=a_{13} a_{24}=a_{14} a_{23}=0, \\
& a_{12}^{2}+a_{34}^{2}=a_{13}^{2}+a_{24}^{2}=a_{23}^{2}+a_{14}^{2} .
\end{aligned}
$$

Moreover, since $0=\Gamma(A)-\Gamma\left(\left[A, E_{12}-E_{21}\right]\right)=a_{12}^{2}+a_{34}^{2}$, we see that $A=0$.

Case $3(n=3)$. Since $0=\Gamma(A)=a_{12}^{2}+a_{23}^{2}+a_{13}^{2}$ and $\Gamma\left(\left[A, E_{12}-\right.\right.$ $\left.\left.E_{21}\right]\right)=a_{23}^{2}+a_{13}^{2}$, it follows that $a_{12}=0$. Similarly $a_{23}=a_{13}=$ 0 .

Lemma 16. Let $y$ be a nonzero element of $\operatorname{nil}\left(K_{[0]} \cap K_{\overline{0}}\right)$. Then there exists $z \in K_{[0]} \cap K_{\overline{0}}$ such that $\llbracket y, z \rrbracket$ is not ad-nilpotent.

Proof. By Lemma 13, we can suppose that

$$
y=\sum_{l \in J_{0}} a_{l} x^{\left(2 \varepsilon_{l}\right)}+\sum_{l, t \in J_{0}, l<t} b_{l t} x_{l} x_{t}+\sum_{l, t \in Y_{1}, l<t} c_{l t} x_{l} x_{t},
$$

where $a_{l}, b_{l t}, c_{l t} \in \mathbb{F}$. Three cases arise as follows.

Case 1. There exists some $a_{i} \neq 0$. Let $z=x^{\left(2 \varepsilon_{i^{\prime}}\right)}$. Then $y, z=$ $\sigma(i) a_{i} x_{i} x_{i^{\prime}}+h$, where $x_{i}$ does not appear in the expression of $h$. Noting that $(\operatorname{ad} \llbracket y, z \rrbracket)^{\ell}\left(x_{i^{\prime}}\right)=a_{i}^{\ell} x_{i^{\prime}}$ for all $\ell \in \mathbb{N}$, we conclude that $\llbracket y, z \rrbracket$ is not ad-nilpotent.

Case 2. All $a_{l}=0$, and there exists $b_{i j} \neq 0$. Let $z=x_{i} x_{j^{\prime}}$. A direct calculation shows that $\llbracket y, z \rrbracket=\sigma(j) a_{i j} x_{i} x_{i^{\prime}}+$ $h$, where $x_{i}$ does not appear in the expression of $h$. As $(\operatorname{ad} \llbracket y, z \rrbracket)^{\ell}\left(x_{i^{\prime}}\right)=\sigma(i)^{\ell} \sigma(j)^{\ell} a_{i j}^{\ell} x_{i^{\prime}}$ and $\forall \ell \in \mathbb{N}$, we see that $\llbracket y, z \rrbracket$ is not ad-nilpotent.

Case 3. All $a_{l}=0$ and $b_{l t}=0$. So $y \in \operatorname{span}_{\mathbb{F}}\left\{x_{i} x_{j} \mid i, j \in Y_{1}\right\}$. This can happen only if $n \geq 3$ by Lemma 13(4). Note that $\rho(y)$ is an antisymmetric nilpotent matrix. Then Lemma 15 provides an element $z$ of $\operatorname{span}_{\mathbb{F}}\left\{x_{i} x_{j} \mid i, j \in Y_{1}\right\}$ such that $\llbracket \rho(y), \rho(z) \rrbracket$ is not a nilpotent matrix. Hence $\llbracket y, z \rrbracket$ is not adnilpotent.

\section{Proofs of Theorems}

Proof of Theorem 1. We proceed in several steps. 
(I) $\operatorname{Nil}\left(K_{\overline{0}}\right)=\operatorname{Nil}\left(K_{0} \cap K_{\overline{0}}\right)$. Suppose that $y$ is an arbitrary element of $\operatorname{nil}\left(K_{\overline{0}}\right)$. It follows from (2) and (3) of Lemma 5 that $y \in K_{0}$, which combined with $y \in \operatorname{nil}\left(K_{\overline{0}}\right)$ yields $y \in$ $\operatorname{nil}\left(K_{0} \cap K_{\overline{0}}\right)$, thus proving $\operatorname{nil}\left(K_{\overline{0}}\right) \subseteq \operatorname{nil}\left(K_{0} \cap K_{\overline{0}}\right)$. Hence $\operatorname{Nil}\left(K_{\overline{0}}\right) \subseteq \operatorname{Nil}\left(K_{0} \cap K_{\overline{0}}\right)$.

The reverse inclusion is clear.

(II) $K_{0} \cap K_{\overline{0}}=\operatorname{Nor}_{K_{\overline{0}}}\left(\operatorname{Nil}\left(K_{\overline{0}}\right)\right)$. We first prove $K_{0} \cap K_{\overline{0}} \subseteq$ $\operatorname{Nor}_{K_{\overline{0}}}\left(\operatorname{Nil}\left(K_{\overline{0}}\right)\right)$. It follows from (I) that

$$
\operatorname{Nor}_{K_{\overline{0}}}\left(\operatorname{Nil}\left(K_{\overline{0}}\right)\right)=\operatorname{Nor}_{K_{\overline{0}}}\left(\operatorname{Nil}\left(K_{0} \cap K_{\overline{0}}\right)\right) .
$$

Therefore

$$
\operatorname{Nil}\left(K_{0} \cap K_{\overline{0}}\right) \subseteq \operatorname{Nor}_{K_{\overline{0}}}\left(\operatorname{Nil}\left(K_{0} \cap K_{\overline{0}}\right)\right)=\operatorname{Nor}_{K_{\overline{0}}}\left(\operatorname{Nil}\left(K_{\overline{0}}\right)\right) .
$$

By formula (12), we see that $\llbracket x_{m}, K_{[0]} \rrbracket=0$ and $\llbracket x_{m}, K_{1} \cap$ $K_{\overline{0}} \rrbracket \subseteq K_{1} \cap K_{\overline{0}}$, proving

$$
x_{m} \in \operatorname{Nor}_{K_{\overline{0}}}\left(\operatorname{Nil}\left(K_{\overline{0}}\right)\right) .
$$

In the case of $n \geq 3$, by (3) and (5) of Lemma 13, we obtain $K_{0} \cap K_{\overline{0}}=\operatorname{Nil}\left(K_{0} \cap K_{\overline{0}}\right)+\mathbb{F} x_{m}$. By (69) and (70), we have $K_{0} \cap K_{\overline{0}} \subseteq \operatorname{Nor}_{K_{\overline{0}}}\left(\operatorname{Nil}\left(K_{\overline{0}}\right)\right)$. In the case of $n=2$, by (4) and (5) of Lemma 13, we have $K_{0} \cap K_{\overline{0}}=\operatorname{Nil}\left(K_{0} \cap K_{\overline{0}}\right)+\mathbb{F} x_{m}+\mathbb{F} x_{m+1} x_{s}$. Note that $x_{m+1} x_{s} \in \operatorname{Nor}_{K_{\overline{0}}}\left(\operatorname{Nil}\left(K_{\overline{0}}\right)\right)$, which combined with (69) and (70) yields $K_{0} \cap K_{\overline{0}} \subseteq \operatorname{Nor}_{K_{\overline{0}}}\left(\operatorname{Nil}\left(K_{\overline{0}}\right)\right)$. In the case of $n=1$, by (4) and (5) of Lemma 13, we have $K_{0} \cap K_{\overline{0}}=$ $\operatorname{Nil}\left(K_{0} \cap K_{\overline{0}}\right)+\mathbb{F} x_{m}$. Hence $K_{0} \cap K_{\overline{0}} \subseteq \operatorname{Nor}_{K_{\overline{0}}}\left(\operatorname{Nil}\left(K_{\overline{0}}\right)\right)$ by $(69)$ and (70).

Conversely, suppose that $y=y_{[-2]}+y_{-1} \in$ $\operatorname{Nor}_{K_{\overline{0}}}\left(\operatorname{Nil}\left(K_{\overline{0}}\right)\right)$, where $y_{[-2]} \in K_{[-2]}, y_{-1} \in K_{-1}$. If $y_{[-2]} \neq 0$, then $\llbracket y, x_{1} x_{m} \rrbracket=2 y_{[-2]} x_{1}+h \notin \operatorname{Nil}\left(K_{\overline{0}}\right)$ by Lemma 13(5), contradicting $y \in \operatorname{Nor}_{K_{\overline{0}}}\left(\operatorname{Nil}\left(K_{\overline{0}}\right)\right)$, where $h \in K_{0}$. Hence $y_{[-2]}=0$ and we can write $y=y_{[-1]}+y_{0}$, where $y_{[-1]}=$ $\sum_{i \in J_{0}} a_{i} x_{i} \in K_{[-1]}, a_{i} \in \mathbb{F}, y_{0} \in K_{0}$. If there is some $a_{j} \neq 0$, then $\llbracket y, x^{\left(2 \varepsilon_{j^{\prime}}\right)} \rrbracket=\sigma(j) a_{j} x_{j^{\prime}}+h$, where $h \in K_{0}$. Lemma 13(5) shows that $\llbracket y, x^{\left(2 \varepsilon_{j^{\prime}}\right)} \rrbracket \notin \operatorname{Nil}\left(K_{\overline{0}}\right)$, contradicting $y \in \operatorname{Nor}_{K_{\overline{0}}}\left(\operatorname{Nil}\left(K_{\overline{0}}\right)\right)$. Hence $y_{[-1]}=0$ and $y \in K_{0} \cap K_{\overline{0}}$, proving $\operatorname{Nor}_{K_{\overline{0}}}\left(\operatorname{Nil}\left(K_{\overline{0}}\right)\right) \subseteq$ $K_{0} \cap K_{\overline{0}}$.

(III) Let $M=\left\{y \in \operatorname{nil}\left(K_{\overline{0}}\right) \mid \llbracket y, K_{0} \cap K_{\overline{0}} \rrbracket \subseteq \operatorname{nil}\left(K_{\overline{0}}\right)\right\}$. Then $M=K_{1} \cap K_{\overline{0}}$. Suppose that $y=y_{[0]}+y_{1}$ is an arbitrary element of $M$, where $y_{[0]} \in K_{[0]} \cap K_{\overline{0}}$ and $y_{1} \in K_{1} \cap K_{\overline{0}}$. If $y_{[0]} \neq 0$, since $y_{[0]} \in \operatorname{nil}\left(K_{[0]}\right)$ by Lemma 5(4), then Lemma 16

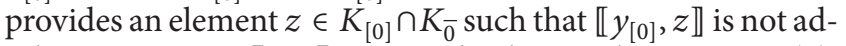
nilpotent. Hence $\llbracket y, z \rrbracket$ is not ad-nilpotent by Lemma 5(4), contradicting $y \in M$. Therefore $y_{[0]}=0$ and $y \in K_{1} \cap K_{\overline{0}}$, thus proving $M \subseteq K_{1} \cap K_{\overline{0}}$.

On the other hand, since $\llbracket K_{1} \cap K_{\overline{0}}, K_{0} \cap K_{\overline{0}} \rrbracket \subseteq K_{1} \cap K_{\overline{0}} \subseteq$ $\operatorname{nil}\left(K_{0} \cap K_{\overline{0}}\right)$ by Lemma 9, it follows that $K_{1} \cap K_{\overline{0}} \subseteq M$.

(IV) Let $Q=\left\{y \in K_{\overline{1}} \mid \llbracket y, K_{\overline{1}} \rrbracket \subseteq K_{0} \cap K_{\overline{0}}\right\}$. Then $Q=$ $K_{1} \cap K_{\overline{1}}$. Suppose that $y=y_{[-1]}+y_{0} \in Q$, where $y_{[-1]}=$ $\sum_{i \in Y_{1}} a_{i} x_{i} \in K_{[-1]} \cap K_{\overline{1}}$ and $y_{0} \in K_{0} \cap K_{\overline{1}}$. If there is some $a_{j} \neq 0$, then $\llbracket y, x_{j} \rrbracket=-a_{j}+\llbracket y_{0}, x_{j} \rrbracket \notin K_{-1} \cap K_{\overline{0}}$, and hence $\llbracket y, x_{j} \rrbracket \notin K_{0} \cap K_{\overline{0}}$, contradicting $y \in Q$. Therefore $y_{[-1]}=0$, and we can write $y=y_{[0]}+y_{1}$, where $y_{[0]}=\sum_{i \in J_{0}, j \in Y_{1}} a_{i j} x_{i} x_{j} \epsilon$ $K_{[0]} \cap K_{\overline{1}}$ and $y_{1} \in K_{1} \cap K_{\overline{1}}$. If there exists some $a_{l t} \neq 0$, then $\llbracket y, x_{t} \rrbracket=-\sum_{i \in J_{0}} a_{i t} x_{i}+\llbracket y_{1}, x_{t} \rrbracket \notin K_{0} \cap K_{\overline{0}}$, a contradiction which yields $y_{[0]}=0$ and $y \in K_{1} \cap K_{\overline{1}}$.
The reverse inclusion follows from the fact that $K_{\overline{1}} \subset K_{-1}$. (V) $\llbracket K_{\overline{1}}, K_{1} \cap K_{\overline{0}} \rrbracket=K_{0} \cap K_{\overline{1}}$. It suffices to show that $K_{0} \cap K_{\overline{1}} \subseteq \llbracket K_{\overline{1}}, K_{1} \cap K_{\overline{0}} \rrbracket$. Suppose that $x^{(\alpha)} x^{u}$ is an arbitrary basis element of $K_{0} \cap K_{\overline{1}}$ with $x^{u}=x_{i} x^{v}$. Note that $x^{\left(\alpha+\varepsilon_{m}\right)} x^{v} \in$ $K_{1} \cap K_{\overline{0}}$. Since $x^{(\alpha)} x^{u}=\llbracket x_{i}, x^{\left(\alpha+\varepsilon_{m}\right)} x^{v} \rrbracket$, it follows that $x^{(\alpha)} x^{u} \in$ $\llbracket K_{\overline{1}}, K_{1} \cap K_{\overline{0}} \rrbracket$, as desired.

It follows from (II) and (V) that $K_{0}=K_{0} \cap K_{\overline{0}}+K_{0} \cap K_{\overline{1}}$ is invariant under automorphisms of $K$. By (III) and (IV), we obtain that $K_{1}=K_{1} \cap K_{\overline{0}}+K_{1} \cap K_{\overline{1}}$ is invariant. Therefore $K_{-1}=\left\{x \in K \mid \llbracket x, K_{1} \rrbracket \subseteq K_{0}\right\}$ is invariant. By the transitivity of $K$, we conclude that

$$
K_{i+1}=\left\{x \in K_{i} \mid \llbracket x, K_{-1} \rrbracket \subseteq K_{i}\right\}, \quad \forall i \geq 0 .
$$

Hence the natural filtration of $K$ is invariant under automorphisms of $K$.

Proof of Theorem 2. Let $\varphi: K(2 r+1, n) \rightarrow K\left(2 r^{\prime}+\right.$ $\left.1, n^{\prime}\right)$ be an isomorphism of Lie superalgebras. Let $K$ and $K^{\prime}$ denote $K(2 r+1, n)$ and $K\left(2 r^{\prime}+1, n^{\prime}\right)$, respectively. Since $\varphi\left(K_{\overline{0}}\right)=K_{\overline{0}}^{\prime}$ and $\varphi\left(\operatorname{Nil}\left(K_{\overline{0}}\right)\right)=\operatorname{Nil}\left(K_{\overline{0}}^{\prime}\right)$, it follows that $\varphi\left(\operatorname{Nor}_{K_{\overline{0}}}\left(\operatorname{Nil}\left(K_{\overline{0}}\right)\right)\right)=\operatorname{Nor}_{K_{\overline{0}}^{\prime}}\left(\operatorname{Nil}\left(K_{\overline{0}}^{\prime}\right)\right)$. By (II) in the proof of Theorem 1, we have

$$
\begin{aligned}
\varphi\left(K_{0} \cap K_{\overline{0}}\right) & =\varphi\left(\operatorname{Nor}_{K_{\overline{0}}}\left(\operatorname{Nil}\left(K_{\overline{0}}\right)\right)\right) \\
& =\operatorname{Nor}_{K_{\overline{0}}^{\prime}}\left(\operatorname{Nil}\left(K_{\overline{0}}^{\prime}\right)\right)=K_{0}^{\prime} \cap K_{\overline{0}}^{\prime} .
\end{aligned}
$$

Consequently

$$
\begin{aligned}
\varphi\left(\left\{y \in \operatorname{nil}\left(K_{\overline{0}}\right) \mid \llbracket y, K_{0} \cap K_{\overline{0}} \rrbracket \subseteq \operatorname{nil}\left(K_{\overline{0}}\right)\right\}\right) \\
\quad=\left\{y \in \operatorname{nil}\left(K_{\overline{0}}^{\prime}\right) \mid \llbracket y, K_{0}^{\prime} \cap K_{\overline{0}}^{\prime} \rrbracket \subseteq \operatorname{nil}\left(K_{\overline{0}}^{\prime}\right)\right\} .
\end{aligned}
$$

Applying (III) in the proof of Theorem 1 , we see that $\varphi\left(K_{1} \cap\right.$ $\left.K_{\overline{0}}\right)=K_{1}^{\prime} \cap K_{\overline{0}}^{\prime}$, which combined with $(\mathrm{V})$ yields

$$
\begin{aligned}
\varphi\left(K_{0} \cap K_{\overline{1}}\right) & =\varphi\left(\llbracket K_{\overline{1}}, K_{1} \cap K_{\overline{0}} \rrbracket\right)=\llbracket K_{\overline{1}}^{\prime}, K_{1}^{\prime} \cap K_{\overline{0}}^{\prime} \rrbracket \\
& =K_{0}^{\prime} \cap K_{\overline{1}}^{\prime} .
\end{aligned}
$$

It follows from (72) and (74) that $\varphi\left(K_{0}\right)=K_{0}^{\prime}$. Therefore $\varphi$ induces an isomorphism of $\mathbb{Z}_{2}$-graded spaces $\widetilde{\varphi}: K / K_{0} \rightarrow$ $K^{\prime} / K_{0}^{\prime}$. A comparison of dimensions shows that $r=r^{\prime}$ and $n=n^{\prime}$. The converse implication is clear.

Proof of Theorem 3. It suffices to prove that $\left.\phi\right|_{K_{[-1]}}=\left.\psi\right|_{K_{[-1]}}$ implies that $\phi=\psi$. Since $\varphi(1)=\varphi\left(\llbracket x_{1}, x_{1^{\prime}} \rrbracket\right) \stackrel{=}{=}$ $\psi\left(\llbracket x_{1}, x_{1^{\prime}} \rrbracket\right)=\psi(1)$, it follows that $\left.\varphi\right|_{K_{[-2]}}=\left.\psi\right|_{K_{[-2]}}$. We use induction on $\ell$ to show that

$$
\left.\varphi\right|_{K_{[\ell]}}=\left.\psi\right|_{K_{[\ell]}}, \quad \forall \ell \geq-1 .
$$

Assume that $\ell \geq 0$ and (75) holds for $\ell-1$. Suppose that $y \in K_{[\ell]}$ and let $z=\varphi(y)-\psi(y)$. We want to prove $z=$ 0 . The induction hypothesis yields that $\varphi\left(x_{i}\right)=\psi\left(x_{i}\right)$ and $\varphi\left(\llbracket y, x_{i} \rrbracket\right)=\psi\left(\llbracket y, x_{i} \rrbracket\right), i \in J$. Therefore,

$$
\begin{aligned}
\llbracket z, \varphi\left(x_{i}\right) \rrbracket & =\llbracket \varphi(y)-\psi(y), \varphi\left(x_{i}\right) \rrbracket \\
& =\varphi\left(\llbracket y, x_{i} \rrbracket\right)-\psi\left(\llbracket y, x_{i} \rrbracket\right)=0 .
\end{aligned}
$$


Since $\varphi\left(K_{0}\right)=K_{0}$ and $\varphi\left(K_{-1}\right)=K_{-1}$ by Theorem 1 , $\varphi$ induces an automorphism $\widetilde{\varphi}$ of the $\mathbb{Z}_{2}$-graded space $K_{-1} / K_{0}$. Consequently there exists a homogeneous basis $\left\{h_{1}, \ldots, h_{m-1}, h_{m+1}, \ldots, h_{s}\right\}$ of $K_{[-1]}$ such that $\left(x_{i}\right) \equiv h_{i}(\bmod$ $\left.K_{0}\right)$. Thus there exist $g_{i} \in K_{0}$ such that $\varphi\left(x_{i}\right)=h_{i}-g_{i}, i \in J$. Therefore, (76) shows that $\llbracket z, h_{i} \rrbracket=\llbracket z, g_{i} \rrbracket$ for all $i \in J$. As $z=\varphi(y)-\psi(y) \in K_{0}$ by Theorem 1 , it can be decomposed into $z=\sum_{j=0}^{t} z_{[j]}$, where $z_{[j]} \in K_{[j]}$. Noting that $\llbracket z_{[0]}, h_{i} \rrbracket \in K_{[-1]}$ and $\llbracket z, g_{i} \rrbracket \in K_{0}$, we obtain $\llbracket z_{[0]}, h_{i} \rrbracket=0$ for all $i \in J$, and hence $z_{[0]}=0$ since $K$ is transitively graded. By induction, we conclude that $z_{[j]}=0, j=0,1, \ldots, t$. Hence, $z=0$, as desired.

\section{Conflict of Interests}

The author declares that there is no conflict of interests regarding the publication of this paper.

\section{References}

[1] R. E. Block and R. L. Wilson, "Classification of the restricted simple Lie algebras," Journal of Algebra, vol. 114, no. 1, pp. 115259,1988

[2] H. Strade, "The classification of the simple modular Lie algebras. IV. Determining the associated graded algebra," Annals of Mathematics, vol. 138, no. 1, pp. 1-59, 1993.

[3] V. G. Kac, "Lie superalgebras," Advances in Mathematics, vol. 26, no. 1, pp. 8-96, 1977.

[4] M. Scheunert, The theory of Lie superalgebras, vol. 716 of Lecture Notes in Mathematics, Springer, Berlin, Germany, 1979.

[5] V. G. Kac, "Description of filtered Lie algebras with which graded Lie algebras of Cartan type are associated," Izvestiya Akademii Nauk SSSR. Seriya Matematicheskaya, vol. 8, no. 4, article 801, 1974.

[6] A. I. Kostrikin and I. R. Shafarevic, "Graded Lie algebras of finite characteristic," Mathematics of the USSR-Izvestiya, vol. 3, no. 2, pp. 237-304, 1969.

[7] N. Jin, "ad-nilpotent elements, quasi-nilpotent elements and invariant filtrations of infinite-dimensional Lie algebras of Cartan type," Science in China, vol. 35, no. 10, pp. 1191-1200, 1992.

[8] Y. Zhang and J. Nan, "Finite-dimensional Lie superalgebras $W(m, n, \underline{t})$ and $S(m, n, \underline{t})$ of Cartan type," Advances in Mathematics, vol. 27, no. 3, pp. 240-246, 1998.

[9] Y. Zhang and H. Fu, "Finite-dimensional Hamiltonian Lie superalgebra," Communications in Algebra, vol. 30, no. 6, pp. 2651-2673, 2002.

[10] L. Ma, L. Chen, and Y. Zhang, "Finite-dimensional simple modular Lie superalgebra $M$," Frontiers of Mathematics in China, vol. 8, no. 2, pp. 411-441, 2013.

[11] X. Xu, L. Chen, and Y. Zhang, "On the modular Lie superalgebra

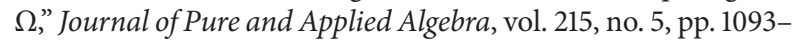
1101, 2011.

[12] K. Zheng and Y. Zhang, "The natural filtration of finite dimensional modular Lie superalgebras of special type," Abstract and Applied Analysis, vol. 2013, Article ID 891241, 7 pages, 2013.

[13] V. G. Kac, "Classification of infinite-dimensional simple linearly compact Lie superalgebras," Advances in Mathematics, vol. 139, no. 1, pp. 1-55, 1998.
[14] Y. Zhang, "Finite-dimensional Lie superalgebras of Cartan type over fields of prime characteristic," Chinese Science Bulletin, vol. 42, no. 9, pp. 720-724, 1997.

[15] Q. Mu and Y. Zhang, "Infinite-dimensional modular special odd contact superalgebras," Journal of Pure and Applied Algebra, vol. 214, no. 8, pp. 1456-1468, 2010. 


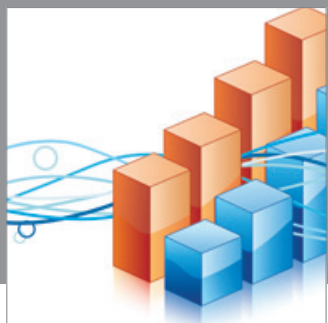

Advances in

Operations Research

mansans

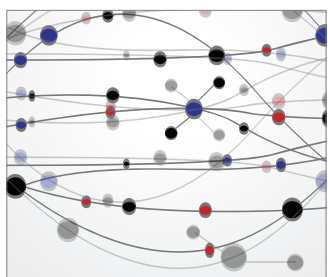

The Scientific World Journal
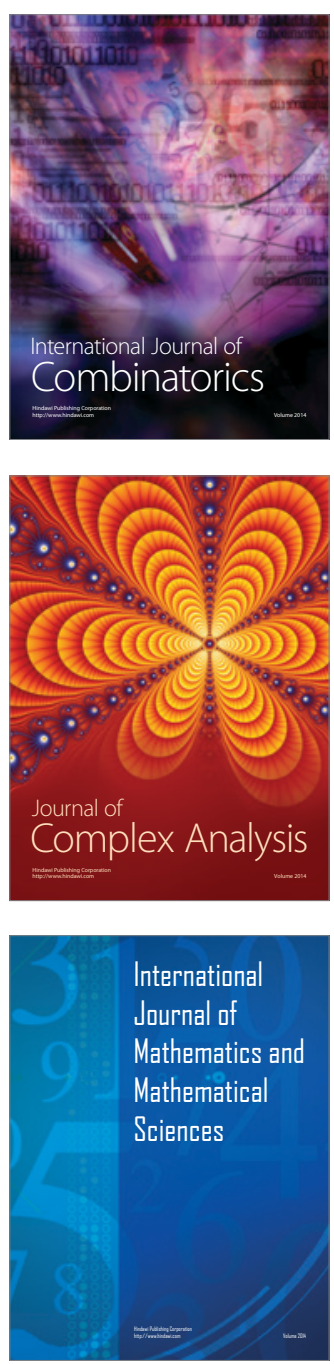
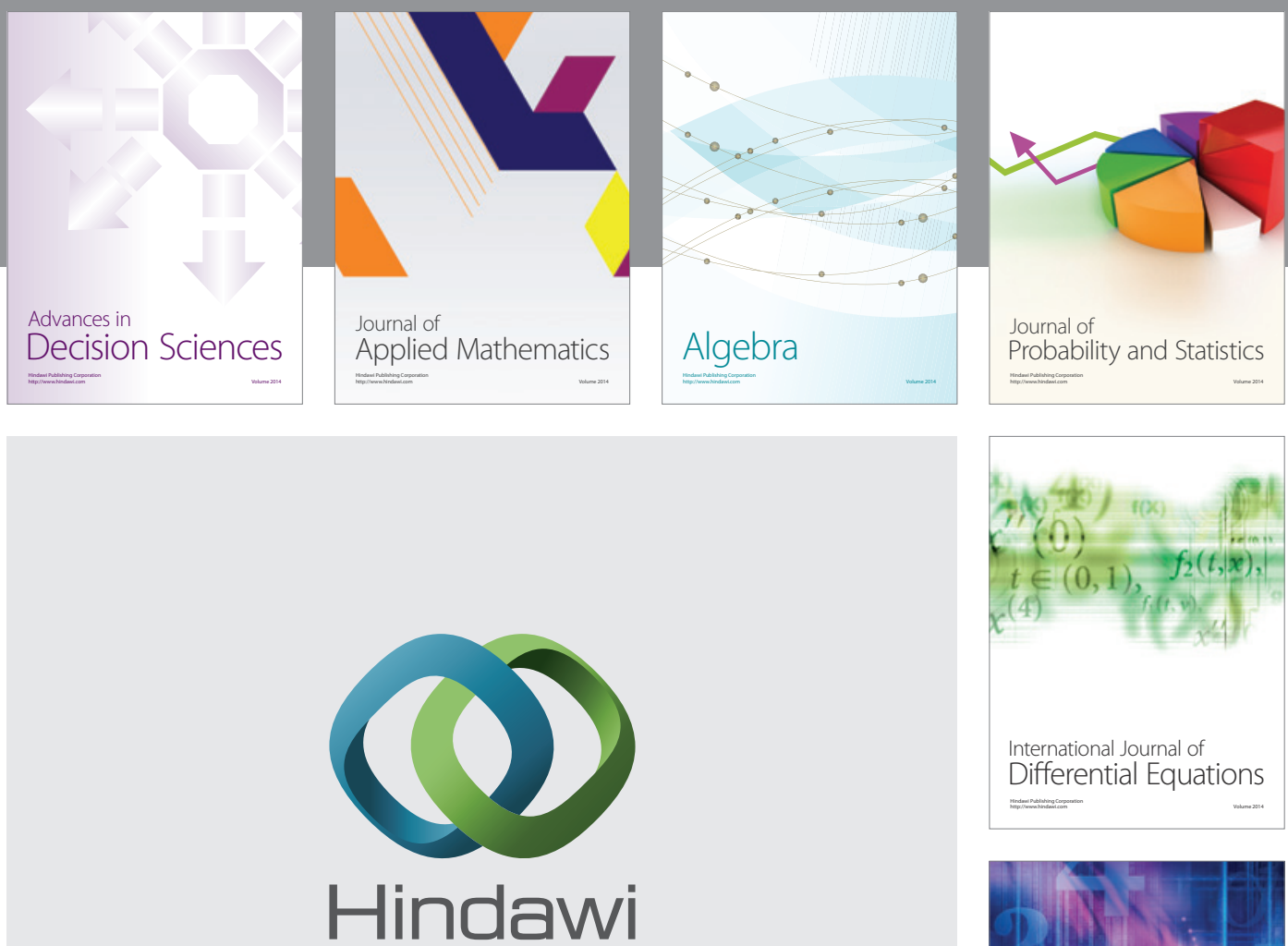

Submit your manuscripts at http://www.hindawi.com
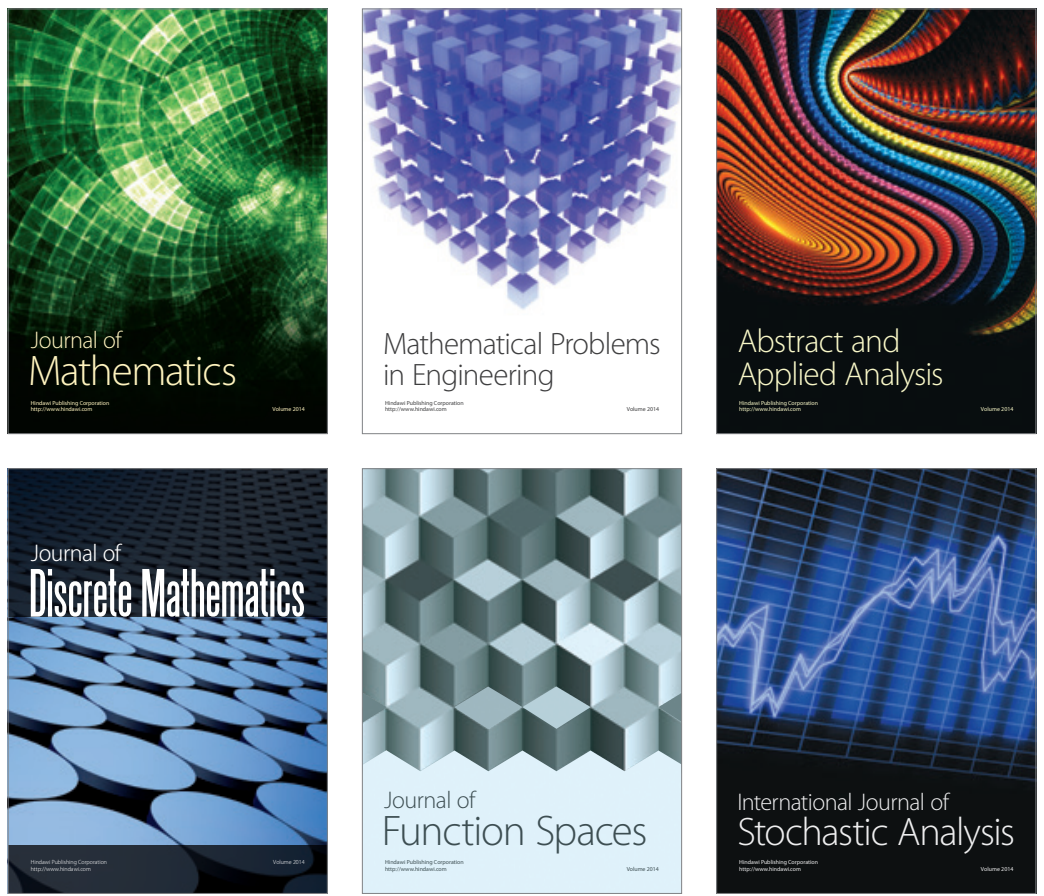

Journal of

Function Spaces

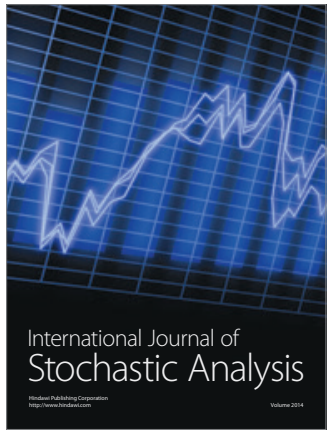

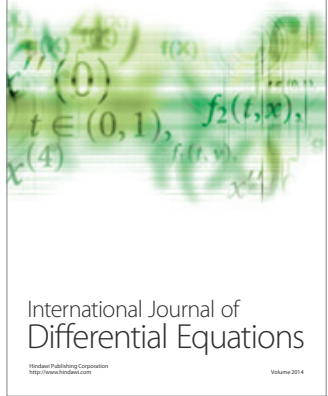
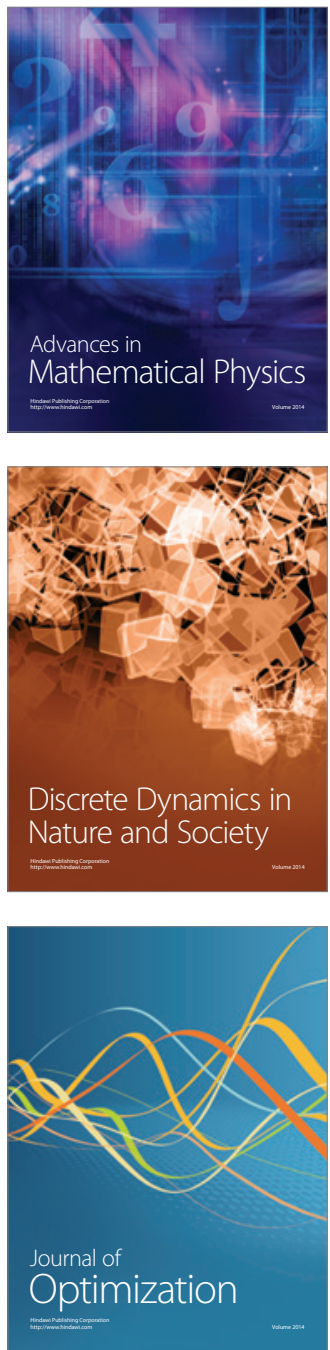